Research Paper

\title{
Nuclear Expression of GS28 Protein: A Novel Biomarker that Predicts Prognosis in Colorectal Cancers
}

\author{
Sung Hak Lee ${ }^{1}$, Hyung Jae Yoo ${ }^{2}$, Do Eun Rim², Yinji Cui ${ }^{3}$, Ahwon Lee ${ }^{1}$, Eun Sun Jung${ }^{1}$, Seung Taek Oh${ }^{4}$, Jun

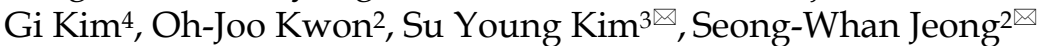 \\ 1. Department of Hospital Pathology, Seoul St. Mary's Hospital, College of Medicine, The Catholic University of Korea, Seoul, Republic of Korea; \\ 2. Department of Biochemistry, College of Medicine, The Catholic University of Korea, Seoul, Republic of Korea; \\ 3. Department of Pathology, College of Medicine, The Catholic University of Korea, Seoul, Republic of Korea; \\ 4. Department of Surgery, Seoul St. Mary's Hospital, College of Medicine, The Catholic University of Korea, Seoul, Republic of Korea.
}

$\triangle$ Corresponding authors: Seong-Whan Jeong MD, PhD, Department of Biochemistry, College of Medicine, The Catholic University of Korea, 222, Banpo-daero, Seocho-gu, Seoul, 06591, Republic of Korea Tel: +82 22258 7291; FAX: +82 2596 4435; E-mail: swjeong@catholic.ac.kr Su Young Kim MD, PhD, Department of Pathology, College of Medicine, The Catholic University of Korea, 222, Banpo-daero, Seocho-gu, Seoul, 06591, Republic of Korea Tel: +82 22258 7315; FAX: +82 2 537-6586; E-mail: suyoung@catholic.ac.kr

(c) Ivyspring International Publisher. This is an open access article distributed under the terms of the Creative Commons Attribution (CC BY-NC) license (https://creativecommons.org/licenses/by-nc/4.0/). See http://ivyspring.com/terms for full terms and conditions.

Received: 2017.01.26; Accepted: 2017.03.23; Published: 2017.04.09

\begin{abstract}
Aims: GS28 (Golgi SNARE protein, $28 \mathrm{kDa}$ ), a member of the soluble $\mathrm{N}$-ethylmaleimide-sensitive factor attachment protein receptors (SNARE) protein family, plays a critical role in mammalian endoplasmic reticulum (ER)-Golgi or intra-Golgi vesicle transport. To date, few researches on the GS28 protein in human cancer tissues have been reported. In this study, we assessed the prognostic value of GS28 in patients with colorectal cancer (CRC).

Methods and results: We screened for GS28 expression using immunohistochemistry in 230 surgical CRC specimens. The CRCs were right-sided and left-sided in $28.3 \%(65 / 230)$ and $71.3 \%$ $(164 / 230)$ of patients, respectively. GS28 staining results were available in 214 cases. Among these, there were 26 nuclear predominant cases and 188 non-nuclear predominant cases. Stromal GS28 expression was noted in 152 cases of CRC. GS28 nuclear predominant immunoreactivity was significantly associated with advanced tumour stage $(p=0.045)$ and marginally associated with perineural invasion $(p=0.064)$. Decreased GS28 expression in the stromal cells was significantly associated with lymph node metastasis $(\mathrm{N}$ stage; $\mathrm{p}=0.036)$. GS28 expression was not associated with epidermal growth factor receptor (EGFR) immunohistochemical positivity or KRAS mutation status. Investigation of the prognostic value of GS28 with Kaplan-Meier analysis revealed a correlation with overall survival $(p=0.004)$. Cases with GS28 nuclear predominant expression had significantly poorer overall survival than those with a non-nuclear predominant pattern.
\end{abstract}

Conclusions: Taken together, these results indicate that GS28 nuclear predominant expression could serve as a prognostic marker for CRC and may help in identifying aggressive forms of CRC.

Key words: GS28 protein, Biologic Marker, Colorectal Carcinoma, Prognosis, Golgi Complex, SNARE proteins.

\section{Introduction}

Colorectal cancer (CRC) is the third most common cancer, and an important contributor to cancer mortality and morbidity worldwide [1]. According to the cancer statistics data of the Ministry of Health and Welfare in Korea, CRC incidence rates in 2012 were 69.3 and 45.9 per 100,000 among men and women, respectively, with rapidly increasing incidence rates in both sexes [2]. Although diagnosis and treatment of CRC have significantly improved over the past two decades, the survival rates in individuals with advanced CRC remain suboptimal, owing to recurrence and metastasis [3]. CRC 
progression is an intricate process associated with cumulative genomic changes [4]. However, underlying mechanisms that control CRC progression and metastasis remain poorly understood. Thus, it is essential to identify proteins regulating CRC progression and metastasis, which will assist in the discrimination of prognostic biomarkers to provide information regarding clinical outcomes of CRC patients, as well as in the development of novel therapeutic targets.

The Golgi apparatus is a polarized organelle, comprising three distinct cisternae: cis, medial, and trans. The Golgi complex functions as a factory in which membrane transport intermediates received from the endoplasmic reticulum (ER) are further processed and sorted for delivery to their eventual destinations: lysosomes, plasma membrane, or secretion [5]. Soluble N-ethylmaleimide-sensitive factor attachment protein receptors (SNAREs) are a group of tail-anchored membrane proteins that play important roles in these membrane trafficking steps. SNAREs on transport vesicles (v-SNAREs) interact with SNAREs on the target membrane (t-SNAREs) in membrane docking and fusion [6]. In mammalian cells, at least 12 different proteins classified as SNAREs were identified in the Golgi [7].

The Golgi apparatus is a platform for molecular signalling between the Golgi and other organelles [8]. Through the organelle networking, the Golgi is involved in crucial roles in cellular activities, including stress sensing, cell death, mitosis checkpoints, and malignant transformation [8]. Numerous proapoptotic/autophagic factors and mitosis-related molecules are localized in the Golgi [9]. Therefore, the Golgi apparatus is becoming increasingly important as an anti-cancer target.

GS28 (Golgi SNARE protein, $28 \mathrm{kDa}$ ) has been described as a member of the SNARE protein family that plays a critical role in mammalian ER-Golgi or intra-Golgi vesicle transport [10, 11]. To date, all reports have focused on the roles of GS28 in vesicular transport, and little is known about the possible roles of this protein in pathological conditions. A recent study demonstrated that deletion mutants of GS28 in C. elegans demonstrated reduced seam cell numbers and a missing ray phenotype during development, suggesting that GS28 has roles in cell proliferation and differentiation [12]. Another report showed that mutations in GS28 lead to retinal degeneration in Drosophila [13]. However, few researches on the GS28 protein in human cancer tissues have yet been reported. We reported very recently that High nuclear expression of GS28 is associated with poor prognosis in cervical cancer patients [14]. The observation suggests the GS28 as a novel prognostic marker in cervical cancers.

Here, we evaluated GS28 expression in CRC in Korean patients. To our knowledge, this is the first study to assess the prognostic value of GS28 in CRC.

\section{Materials and Methods}

\section{Patients and tumour tissues}

A total of 230 patients (140 men and 90 women) with CRC who had undergone surgical procedures at Seoul St. Mary's Hospital, The Catholic University of Korea, between 2008 and 2011 were enrolled in the study. Clinicopathological data were obtained retrospectively from medical records and pathology reports. Patients ranged in age from 32 to 93 (mean, 62.3) years. Mean tumour size was $4.85 \mathrm{~cm}$ (range, 0.7-17.0). The study was approved by the Institutional Review Board of the Catholic University of Korea, College of Medicine (MC14SNSI0093, Oct. 6, 2014).

\section{Tissue microarray construction and immunohistochemistry}

Following review of histologic sections from the 230 cases of CRC, tissue microarrays (TMAs) were constructed from paraffin-embedded blocks with a Manual Tissue Arrayer (Beecher Instruments, Inc., Sun Prairie, WI, USA) with a $2.0-\mathrm{mm}$ tip. The TMA blocks were sectioned at a thickness of $4 \mu \mathrm{m}$, and the sections were transferred to ProbeOn Plus slides (Fisher Scientific, Pittsburgh, PA, USA) and baked for 2 hours in a dry oven at $56^{\circ} \mathrm{C}$ (Agilent Technologies, Santa Clara, CA, USA). Immunohistochemistry using diluted (1:500) anti-GS28 antibody (BD Biosciences, Franklin Lakes, NJ, USA) was performed according to a previously reported protocol [15]. GS28 expression was categorized into 4 grades according to the intensity of nuclear, cytoplasmic, and stromal staining, respectively (0, no stain; 1 , weak; 2 , moderate; 3, strong). Additionally, the authors evaluated CRC according to the differences between nuclear and cytoplasmic staining. Cases in which the nuclear staining score exceeded the cytoplasmic staining score were considered "nuclear predominant", and cases in which the cytoplasmic staining score exceeded the nuclear staining score, or cases with equal scores for nuclear and cytoplasmic staining, were considered "non-nuclear predominant". Positivity for EGFR expression was defined as $>10 \%$ of tumour cells with any membrane staining above the background level. Cytoplasmic staining without associated membrane staining was considered negative, as in our previous study [16]. Immunohistochemical staining was independently examined by 2 pathologists (S. H. Lee and E. S. Jung). 


\section{KRAS mutation test}

Genomic DNA was isolated from formalin-fixed, paraffin-embedded tissue sections at a thickness of $10 \mu \mathrm{m}$, containing a representative tumour-rich area, with the QIAamp DNA Mini Kit (Qiagen, Hilden, Germany). Tumour areas were manually microdissected from glass slides with a scalpel under a dissecting microscope in a subset of samples. We performed mutational analysis of exons 2 and 3 of KRAS genes using a previously described extraction method [17].

\section{Statistical analysis}

The chi-square or Fisher's exact test was used to assess the association between GS28 expression and various clinicopathological parameters and molecular markers. The survival rate was calculated with the Kaplan-Meier method and differences were evaluated using the log-rank test. In all tests, two-sided P values $<0.05$ were considered statistically significant. Data were analysed using the SPSS statistical software version 21.0 (IBM Corp., Armonk, NY, USA) for Windows.

\section{Results}

\section{Patient characteristics}

In the 230 patients who underwent operation, masses were right-sided and left-sided in $28.3 \%$ $(65 / 230)$ and $71.3 \%(164 / 230)$ of patients, respectively. In one case, no information was available regarding the tumour site. Histologic examinations revealed $216(93.9 \%)$ adenocarcinomas, $10(4.3 \%)$ mucinous adenocarcinomas, and 4 other tumours. Patient characteristics and clinicopathological features are summarized in Table 1.

\section{Association of GS28 expression with clinicopathological features and molecular markers}

In the normal colorectal mucosa, GS28 is expressed in the cytoplasm of the crypt epithelium with weak to moderate intensity (Figure 1A). In the CRC tissues, GS28 staining results were available in 214 cases. GS28 immunoreactivity was revealed in 213 cases (99.5\%) of CRC. Among these, 28 cases showed weak immunopositivity, and 92 and 93 cases showed moderate and strong staining, respectively (Figure 1B-1D). There were 26 nuclear predominant cases and 188 non-nuclear predominant cases (Figure 2A and 2B). Stromal GS28 expression was demonstrated in 152 cases of CRC.

GS28 nuclear predominant immunoreactivity was significantly associated with advanced tumour stage (T stage; $p=0.045)$ and marginally associated with perineural invasion $(p=0.064)$ (Table 2$)$. Other clinicopathological features were not associated with GS28 expression. As stromal cells of tumour tissues are important in the progression of CRC, we evaluated the association of GS28 expression with clinicopathological parameters. Decreased GS28 expression in the stromal cells was significantly associated with lymph nodes metastasis ( $\mathrm{N}$ stage; $\mathrm{p}=$ 0.036) (Table 3). EGFR expression and KRAS mutations are important well-known molecular markers in CRC. However, GS28 expression was not associated with EGFR immunohistochemical positivity or KRAS mutation status in the current study (Tables 4 and 5).

Table 1. Clinicopathological data and molecular marker expression in $230 \mathrm{CRC}$ patients

\begin{tabular}{ll}
\hline Characteristics & $\mathbf{N}(\%)$ \\
\hline Sex & \\
Male & $140(60.9)$ \\
Female & $90(39.1)$ \\
Age & \\
$\leq 55$ years & $60(26.1)$ \\
$>55$ years & $170(73.9)$ \\
Tumour stage ${ }^{a}$ & \\
T1 & $2(0.9)$ \\
T2 & $14(6.1)$ \\
T3 & $157(68.3)$ \\
T4 & $51(22.2)$ \\
Nodal stageb & \\
N0 & $86(37.4)$ \\
N1 & $76(33.0)$ \\
N2 & $64(27.8)$ \\
Metastasis & \\
M0 & $211(91.7)$ \\
M1 & $19(8.3)$ \\
Sitec & \\
Right colon & $65(28.3)$ \\
Left colon & $86(37.4)$ \\
Rectum & $78(33.9)$ \\
\hline a Data regarding tumour stage were unavailable in 6 cases. \\
bData regarding nodal stage were unavailable in 4 cases. \\
cData regarding tumour location were unavailable in 1 case. \\
CRC: colorectal cancer \\
\hline
\end{tabular}

Prognostic values of GS28 expression in CRC

Thirty-one patients expired during the study period. We investigated the prognostic value of GS28 with Kaplan-Meier analysis, and revealed a correlation with overall survival $(p=0.004)$ (Table 6 and Figure 3). Our results showed that the cases with GS28 nuclear predominant expression had significantly poorer overall survival than those with a non-nuclear predominant pattern. Additionally, there were no significant survival differences between CRCs with GS28 stromal expression and non-expression (data not shown). Taken together, these results indicate that GS28 nuclear predominant expression could serve as a prognostic marker for CRC. 

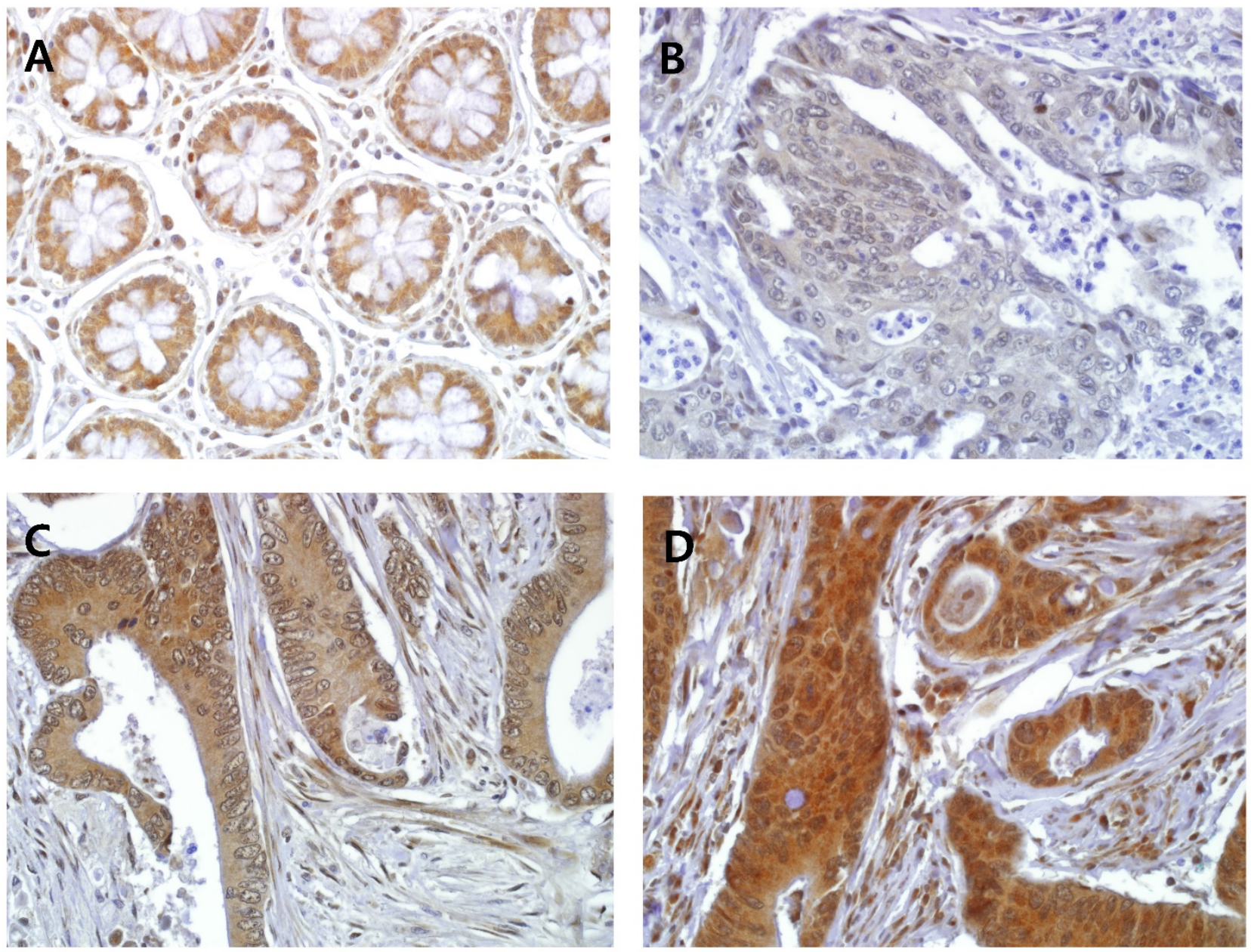

Figure 1. Representative GS28 immunohistochemical staining in (A) normal colonic mucosa and CRC with (B) weak cytoplasmic staining, (C) moderate cytoplasmic staining and (D) strong cytoplasmic staining results $(\times 400)$. Stromal immunoreactivity is also shown in myofibroblastic cells of the lamina propria $(C$ and $D)$.
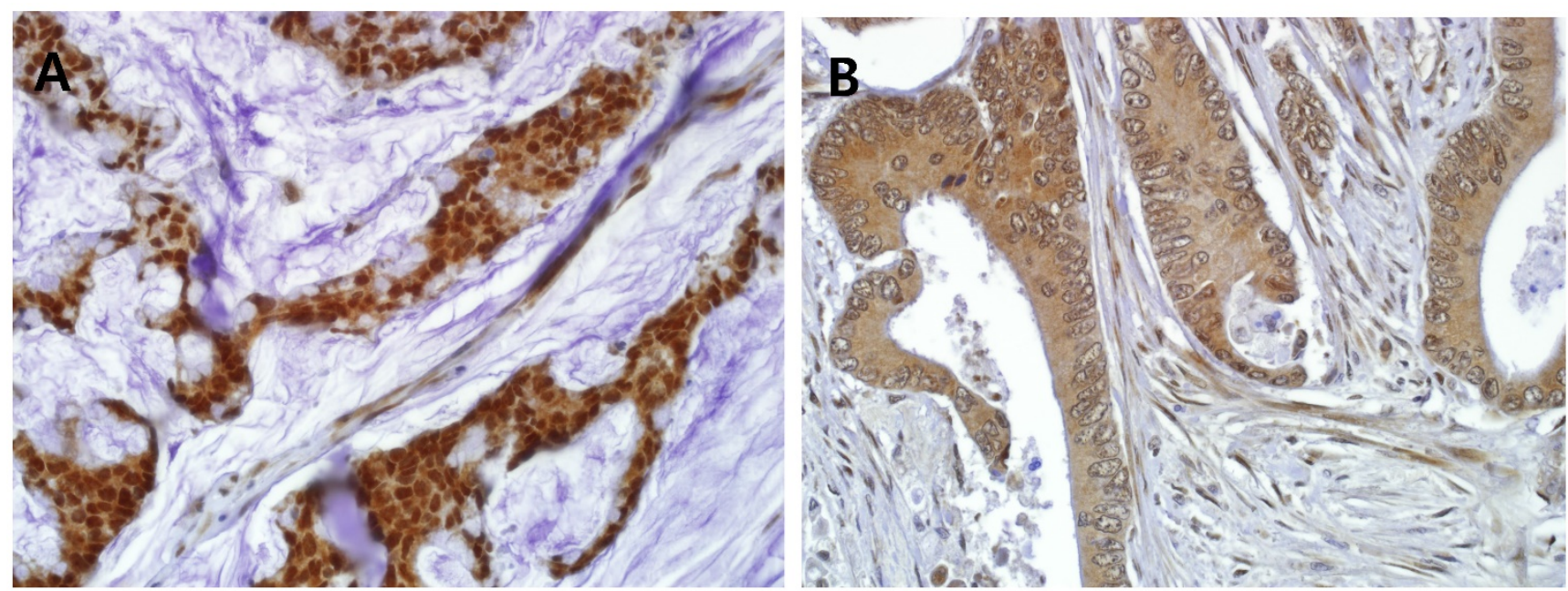

Figure 2. Representative GS28 immunohistochemical staining in CRC with (A) nuclear predominant pattern (nuclear staining: 3, cytoplasmic staining: 1) (B) non-nuclear predominant pattern (nuclear staining: 0, cytoplasmic staining: 2$)(\times 400)$. 
Table 2. Relationship between GS28 expression and clinicopathological parameters in CRC patients

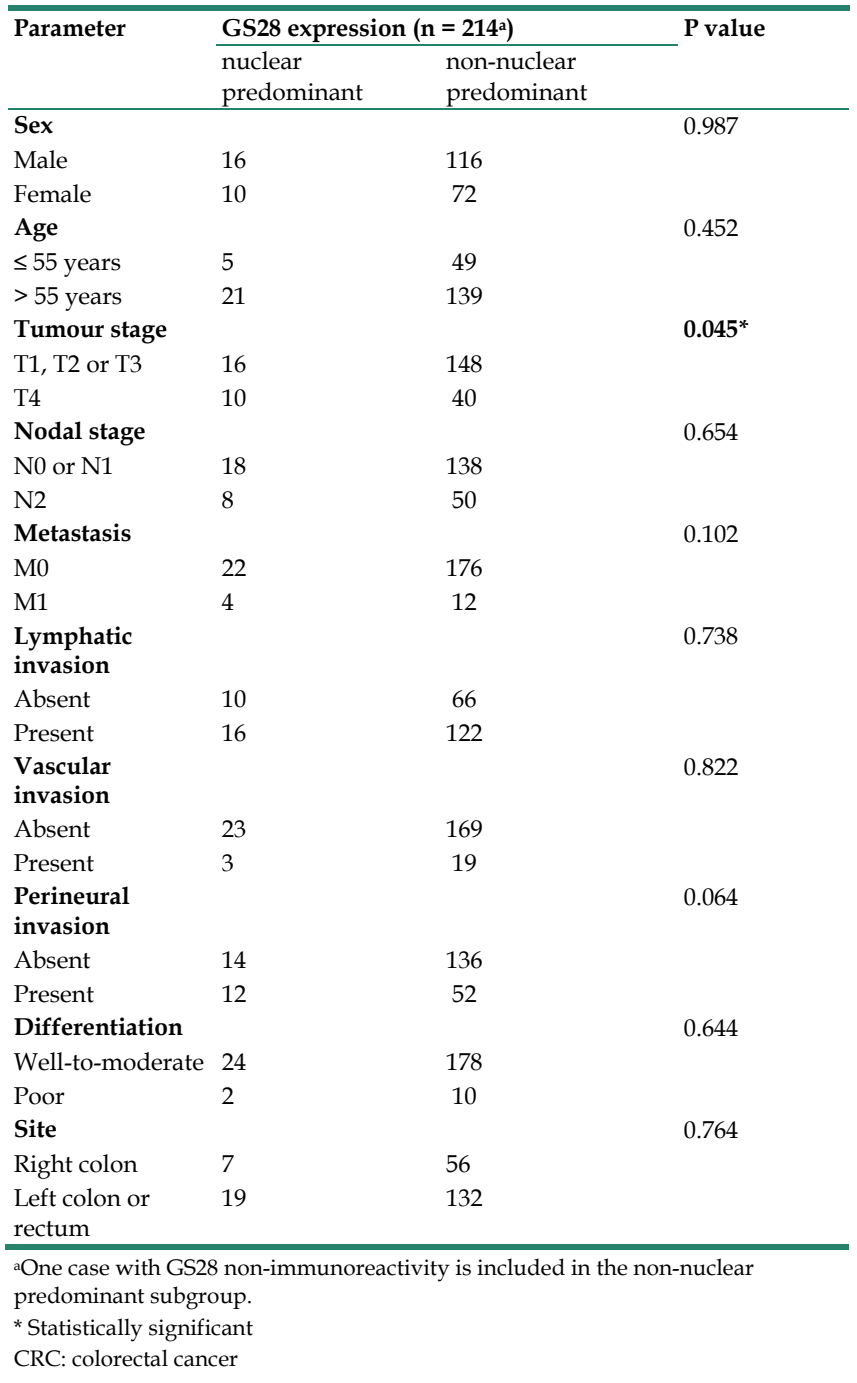

Table 3. Relationship between stromal GS28 expression and the clinicopathological parameters in CRC patients

\begin{tabular}{|c|c|c|c|}
\hline \multirow[t]{2}{*}{ Parameter } & \multicolumn{2}{|c|}{ Stromal GS28 expression $(n=214)$} & \multirow[t]{2}{*}{ Pvalue } \\
\hline & Positive & Negative & \\
\hline Sex & & & 0.487 \\
\hline Male & 96 & 36 & \\
\hline Female & 56 & 26 & \\
\hline Age & & & 0.244 \\
\hline$\leq 55$ years & 35 & 19 & \\
\hline$>55$ years & 117 & 43 & \\
\hline Tumour stage & & & 0.863 \\
\hline $\mathrm{T} 1, \mathrm{~T} 2$ or $\mathrm{T} 3$ & 116 & 48 & \\
\hline $\mathrm{T} 4$ & 36 & 14 & \\
\hline Nodal stage & & & $0.036^{*}$ \\
\hline N0 or N1 & 117 & 39 & \\
\hline N2 & 35 & 23 & \\
\hline Metastasis & & & 0.054 \\
\hline M0 & 144 & 54 & \\
\hline M1 & 8 & 8 & \\
\hline $\begin{array}{l}\text { Lymphatic } \\
\text { invasion }\end{array}$ & & & 0.995 \\
\hline Absent & 54 & 22 & \\
\hline Present & 98 & 40 & \\
\hline $\begin{array}{l}\text { Vascular } \\
\text { invasion }\end{array}$ & & & 0.853 \\
\hline Absent & 136 & 56 & \\
\hline Present & 16 & 6 & \\
\hline $\begin{array}{l}\text { Perineural } \\
\text { invasion }\end{array}$ & & & 0.631 \\
\hline Absent & 108 & 42 & \\
\hline Present & 44 & 20 & \\
\hline Differentiation & & & 1.000 \\
\hline Well-to-moderate & 143 & 59 & \\
\hline Poor & 9 & 3 & \\
\hline Site & & & 0.563 \\
\hline Right colon & 43 & 20 & \\
\hline $\begin{array}{l}\text { Left colon or } \\
\text { rectum }\end{array}$ & 109 & 42 & \\
\hline
\end{tabular}

Table 5. Relationship between stromal GS28 expression and epidermal growth factor receptor expression, and KRAS mutation status in CRC patients

\begin{tabular}{llll}
\hline Marker & \multicolumn{2}{l}{ Stromal GS28 expression $(\mathbf{n}=\mathbf{2 1 4})$} & P value \\
\cline { 2 - 3 } & Positive & Negative & \\
\hline KRAS mutation & & 22 & \\
Positive & 67 & 40 & 0.247 \\
Negative & 85 & 44 & \\
EGFR expression & & 18 & \\
Positive & 115 & 18 & \\
Negative & 37 & & \\
\hline \multicolumn{2}{l}{ EGFR: epidermal growth factor receptor; CRC: colorectal cancer }
\end{tabular}

Table 4. Relationship between GS28 expression and epidermal growth factor receptor expression, and KRAS mutation status in CRC patients

\begin{tabular}{llcl}
\hline Marker & \multicolumn{2}{l}{ GS28 expression $(\mathbf{n}=\mathbf{2 1 4})$} & P value \\
\cline { 2 - 3 } & $\begin{array}{l}\text { nuclear } \\
\text { predominant }\end{array}$ & $\begin{array}{c}\text { non-nuclear } \\
\text { predominant }\end{array}$ & \\
\hline KRAS mutation & & 78 & 0.937 \\
Positive & 11 & 110 & \multirow{2}{*}{0.267} \\
Negative & 15 & & \\
EGFR expression & & 142 & \\
Positive & 17 & 46 & \\
Negative & 9 & 46 & \\
\hline EGFR: epidermal growth factor receptor; CRC: colorectal cancer
\end{tabular}

Table 6. Kaplan-Meier analysis of overall survival in CRC patients

\begin{tabular}{llll}
\hline Variable & \multicolumn{2}{l}{ Kaplan-Meier analysis } & \multirow{2}{*}{ P value } \\
\cline { 2 - 3 } & $\mathrm{M} \pm$ SE (Days) & $95 \%$ CI & \\
\hline $\begin{array}{l}\text { GS28 nuclear } \\
\text { predominant }\end{array}$ & $1881.42 \pm 189.38$ & $1510.24-2252.61$ & \multirow{0}{*}{$\mathbf{0 0 4}^{*}$} \\
$\begin{array}{l}\text { GS28 non-nuclear } \\
\text { predominant }\end{array}$ & $2300.14 \pm 43.42$ & $2215.04-2385.23$ & \\
* Statistically significant & & \\
CRC: colorectal cancer; M: mean; SE: standard error; CI: confidence interval
\end{tabular}




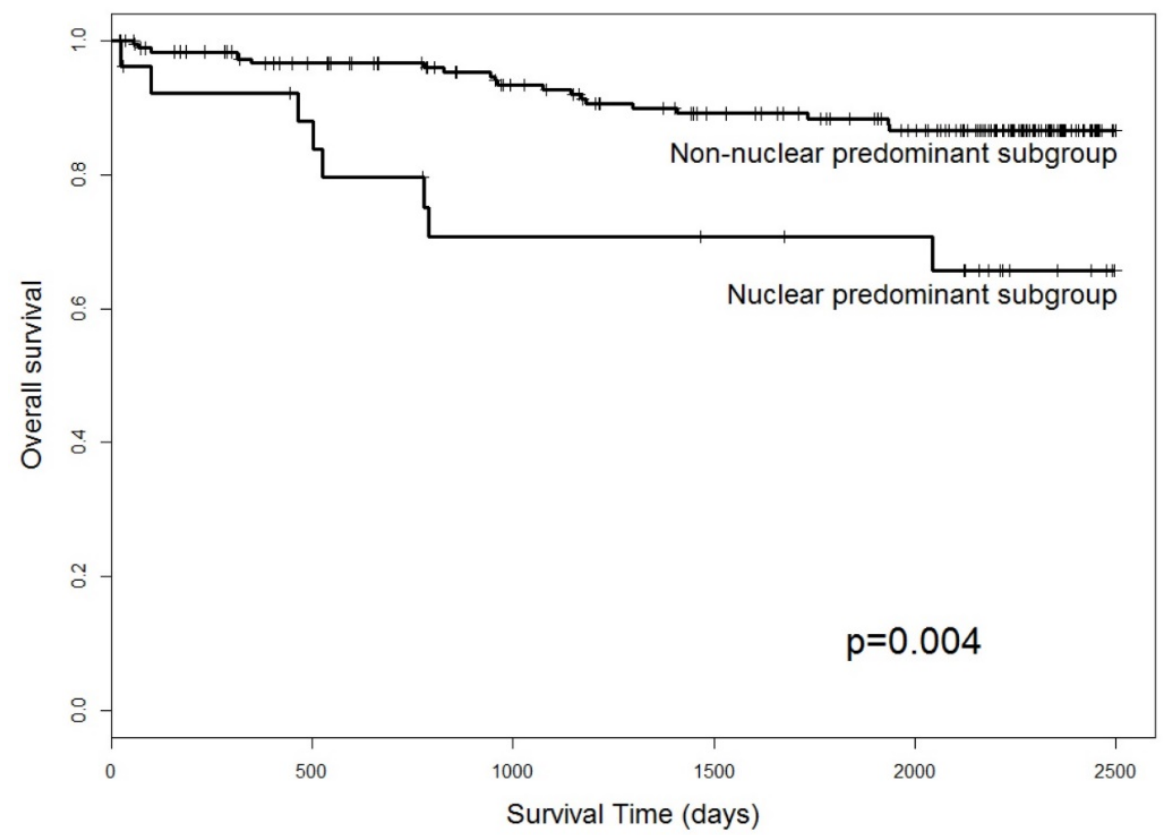

Figure 3. Association of the overall survival of CRC patients with GS28 tumour cell expression. Cases with GS28 nuclear predominant expression showed significantly poorer overall survival.

\section{Discussion}

We found that increased nuclear expression of GS28 in primary CRC tissues significantly correlated with advanced $\mathrm{T}$ stage tumours $(\mathrm{p}=0.045)$, and decreased stromal expression of GS28 significantly correlated with advanced $\mathrm{N}$ stage tumours $(\mathrm{p}=0.036)$. Additionally, increased nuclear GS28 expression was marginally associated with perineural invasion $(\mathrm{p}=$ 0.064 ). We were unable to find an association of cytosolic or nuclear GS28 expression with other clinicopathological parameters, such as sex, M stage, tumour differentiation, EGFR expression, or KRAS mutation. Therefore, a larger-scale study might be necessary to further evaluate the clinicopathological values in CRC tissues. This study is the first to examine the correlation between GS28 expression and clinicopathological parameters in CRC tissues.

The ER and Golgi apparatus are two major organelles that play important roles in the processing, sorting, and transport of newly synthesized secretory and transmembrane proteins [9]. The ER-Golgi network is a hub for various signalling pathways involved in crucial cellular activities, including cell death and malignant transformation [8]. The localization of caspase 2, Polo-like kinase 3 (Plk3), and GD3 synthase to Golgi suggested that the Golgi may be active in the crucial cellular activities [18-20].

GS28 is a $28 \mathrm{kDa}$ membrane protein that appears to play an essential role in intra-Golgi or ER-Golgi vesicle transport [10]. Mammalian SNAREs known to participate in vesicular transport include GS28, Bet1,
Sec22b, and syntaxin 5 [21, 22]. Very few studies have focused on the possible roles of these proteins in pathological conditions, however. Studies in C. elegans and Drosophila GS28 mutants have suggested that GS28 plays important roles in proliferation and differentiation of seam cells and in maintenance of retinal neurons $[12,13]$. We reported previously that GS28 plays a protective role in hydrogen peroxide-induced cell death via inhibition of p38 MAPK in glutathione-depleted neuronal cells [23]. However, few researches to examine GS28 expression in human pathological tissues have yet been reported.

Recent studies have shown that the ER stress-related signalling pathways and malfunction of the Golgi apparatus are involved in cancer development $[9,24]$. The present study demonstrated that increased nuclear expression of GS28 in CRC is significantly correlated with advanced $\mathrm{T}$ stage tumours. Considering that GS28 is a protein located in the Golgi apparatus, it can be speculated that translocation of GS28 into the nuclear compartment may be related to increases in tumour cell migration and invasion, possibly via interactions between GS28-induced nuclear functions and the Golgi apparatus. Syntaxin 17, another SNARE protein, was found to be localized in the cytoplasm, nucleus, and both in several types of cells [25]. Furthermore, its localization was altered in tumour cells compared with their normal counterparts, suggesting that syntaxin 17 may possess additional novel roles in cell proliferation and transformation. We observed nuclear GS28 expression in TMAs of cervical cancer, 
and a significant association between the high nuclear expression of GS28 and the advanced T stage tumors [14]. We, furthermore, demonstrated that patients with high nuclear expression of GS28 showed significantly worse overall survival and progression-free survival, compared to those with low or no nuclear expression. These suggest that the nuclear expression of GS28 protein plays important roles in the progression of CRC. However, molecular mechanisms of protein translocation and its roles remain unknown. Sun et al. [26] revealed that GS28 forms a complex with p53 and its ubiquitin ligase MDM2. They showed that overexpression of GS28 promotes cisplatin-induced apoptosis by reducing the ubiquitination and degradation of p53. In contrast, knockdown of GS28 using shRNA (short hairpin RNA) demonstrated the opposite result in response to cisplatin. These findings offer the first evidence that SNARE proteins can be involved in chemosensitivity, although these results have only been observed in vitro. It has not yet been confirmed that interactions among p53, MDM2, and GS28 proteins occur in the cytosolic or nuclear compartments.

We predicted conserved motifs in the GS28 protein (250 amino acids) using web-based software PROSITE and PredictProtein. Only one hit displayed in the prediction is coiled-coil helices (called SNARE motifs), which mediate the interactions between SNARE proteins. A nuclear localization signal motif is not contained in the GS28 protein. Motifs with high probability of occurrence are glycosylation sites and target sites of phosphorylation for casein kinase II (CKII), protein kinase C (PKC), and CAMP- and cGMP-dependent protein kinases. Involvement of CKII and the tumour promoter PKC as poor prognostic factors in CRC has been reported [27]. However, GS28 phosphorylation and its nuclear localization have not yet been reported. Further studies should be performed to confirm the molecular mechanisms of the protein kinases and the phosphorylation of GS28 in CRC.

It has been shown that $30 \%$ of patients with node-negative CRC on conventional histopathological analysis die from metastatic disease [28]. However, there is no standard method to identify lymphatic and blood vessel invasion, which are reliable independent prognostic factors in patients with node-negative CRC [28]. We identified a reverse relationship between $\mathrm{N}$ stage of CRC and GS28 expression in the stromal fibroblasts. Stromal cells contribute to CRC development and progression via secreting regulatory molecules [29]. Thus, low GS28 expression in stromal fibroblasts might be a prognostic factor for patients with node-negative CRC.

We observed an association trend of increased nuclear GS28 protein with perineural invasion in CRC. The presence of perineural invasion was suggested as an independent prognostic factor for a more aggressive phenotype and poor prognosis in CRC $[30,31]$. Perineural invasion was strongly correlated with high tumour stage, poor differentiation, nodal involvement, infiltrative growth, lymphatic invasion, and venous invasion. Adjuvant therapy was suggested particularly for node-negative CRC patients with perineural invasion [31]. However, further studies with larger populations of CRC patients should be performed to confirm a significant association between GS28 expression and perineural invasion.

Thus, we assessed for the first time the prognostic value of GS28 in colorectal adenocarcinoma. Our findings indicate that GS28 nuclear predominant expression appears to be an independent predictor of poorer survival in patients with CRC. GS28 may be a potential novel candidate for a prognostic biomarker in the battle against CRC. Our study results provide a better understanding of the importance of GS28 in tumour development and may enable the establishment of clinically useful therapeutic targets.

\section{Acknowledgments}

This research was supported by the Basic Science Research Program through the National Research Foundation of Korea (NRF) funded by the Ministry of Education (2013R1A1A2011752).

\section{Author Contributions}

S. H. Lee, S. Y. Kim, and S. W. Jeong designed the research. H. J. Yoo, Y. Cui, S. H. Lee, S. Y. Kim, D. E. Rim, E. S. Jung, and A. Lee performed the experiments. S. T. Oh and J. G. Kim collected the tissues. S. H. Lee, S. Y. Kim, O. J. Kwon, and S.W. Jeong analysed the data. S. H. Lee, S. Y. Kim, and S. $\mathrm{W}$. Jong wrote the paper.

\section{Competing Interests}

The authors have declared that no competing interest exists.

\section{References}

1. Shike M, Winawer SJ, Greenwald PH, Bloch A, Hill MJ, Swaroop SV. Primary prevention of colorectal cancer. The WHO Collaborating Centre for the Prevention of Colorectal Cancer. Bull World Health Organ. 1990; 68: 377-85.

2. Jung KW, Won YJ, Kong HJ, Oh CM, Cho H, Lee DH, et al. Cancer statistics in Korea: incidence, mortality, survival, and prevalence in 2012. Cancer Res Treat. 2015; 47: 127-41.

3. Schmoll HJ, Stein A. Colorectal cancer in 2013: Towards improved drugs, combinations and patient selection. Nat Rev Clin Oncol. 2014; 11: 79-80.

4. Cancer Genome Atlas Network. Comprehensive molecular characterization of human colon and rectal cancer. Nature. 2012; 487: 330-7.

5. Mellman I, Simons K. The Golgi complex: in vitro veritas? Cell. 1992; 68: 829-40.

6. Malsam J, Söllner TH. Organization of SNAREs within the Golgi stack. Cold Spring Harb Perspect Biol. 2011; 3: a005249. 
7. Tai G, Lu L, Wang TL, Tang BL, Goud B, Johannes L, et al. Participation of the syntaxin 5/Ykt6/GS28/GS15 SNARE complex in transport from the early/recycling endosome to the trans-Golgi network. Mol Biol Cell. 2004; 15: 4011-22.

8. Wilson C, Venditti R, Rega LR, Colanzi A, D'Angelo G, De Matteis MA. The Golgi apparatus: an organelle with multiple complex functions. Biochem J. 2011; 433: 1-9.

9. Wlodkowic D, Skommer J, McGuinness D, Hillier C, Darzynkiewicz Z. ER-Golgi network--a future target for anti-cancer therapy. Leuk Res. 2009; 33: 1440-7.

10. Subramaniam VN, Peter F, Philp R, Wong SH, Hong W. GS28, a 28-kilodalton Golgi SNARE that participates in ER-Golgi transport. Science. 1996; 272: 1161-3.

11. Nagahama M, Orci L, Ravazzola M, Amherdt M, Lacomis L, Tempst P, et al. A v-SNARE implicated in intra-Golgi transport. J Cell Biol. 1996; 133: 507-16.

12. Maekawa M, Inoue $T$, Kobuna $H$, Nishimura $T$, Gengyo-Ando $K$, Mitani $S$, et al. Functional analysis of GS28, an intra-Golgi SNARE, in Caenorhabditis elegans. Genes Cells. 2009; 14: 1003-13.

13. Rosenbaum EE, Vasiljevic E, Cleland SC, Flores C, Colley NJ. The Gos 28 SNARE protein mediates intra-Golgi transport of rhodopsin and is required for photoreceptor survival. J Biol Chem. 2014; 289: 32392-409.

14. Cho U, Kim HM, Park HS, Kwon OJ, Lee A, Jeong SW. Nuclear Expression of GS28 Protein: A Novel Biomarker that Predicts Worse Prognosis in Cervical Cancers. PLoS One. 2016; 11: e0162623.

15. Kim YI, Lee A, Lee BH, Kim SY. Prognostic significance of syndecan-1 expression in cervical cancers. J Gynecol Oncol. 2011; 22: 161-7.

16. Lee SH, Lee YS, Hong YG, Kang CS. Significance of COX-2 and VEGF expression in histopathologic grading and invasiveness of meningiomas. APMIS. 2014; 122: 16-24.

17. Kim SY, Choi EJ, Yun JA, Jung ES, Oh ST, Kim JG, et al. Syndecan-1 expression is associated with tumor size and EGFR expression in colorectal carcinoma: a clinicopathological study of 230 cases. Int J Med Sci. 2015; 12: 92-9.

18. Mancini M, Machamer CE, Roy S, Nicholson DW, Thornberry NA, Casciola-Rosen LA, et al. Caspase-2 is localized at the Golgi complex and cleaves golgin-160 during apoptosis. J Cell Biol. 2000; 149: 603-12.

19. Ruan Q, Wang Q, Xie S, Fang Y, Darzynkiewicz Z, Guan K, et al. Polo-like kinase 3 is Golgi localized and involved in regulating Golgi fragmentation during the cell cycle. Exp Cell Res. 2004; 294: 51-9.

20. Rippo MR, Malisan F, Ravagnan L, Tomassini B, Condo I, Costantini P, et al. GD3 ganglioside directly targets mitochondria in a bcl-2-controlled fashion. FASEB J. 2000; 14: 2047-54.

21. Zhang T, Hong W. Ykt6 forms a SNARE complex with syntaxin 5, GS28, and Bet1 and participates in a late stage in endoplasmic reticulum-Golgi transport. J Biol Chem. 2001; 276: 27480-7.

22. Glick BS, Nakano A. Membrane traffic within the Golgi apparatus. Annu Rev Cell Dev Biol. 2009; 25: 113-32.

23. Lee HO, Byun YJ, Cho KO, Kim SY, Lee SB, Kim HS, et al. GS28 Protects Neuronal Cell Death Induced by Hydrogen Peroxide under Glutathione-Depleted Condition. Korean J Physiol Pharmacol. 2011; 15: 149-56.

24. Gong H, Feng L. Computational analysis of the roles of ER-Golgi network in the cell cycle. BMC Syst Biol. 2014; 8 Suppl 4: S3.

25. Zhang Q, Li J, Deavers M, Abbruzzese JL, Ho L. The subcellular localization of syntaxin 17 varies among different cell types and is altered in some malignant cells. J Histochem Cytochem. 2005; 53: 1371-82.

26. Sun NK, Huang SL, Chien KY, Chao CC. Golgi-SNARE GS28 potentiates cisplatin-induced apoptosis by forming GS28-MDM2-p53 complexes and by preventing the ubiquitination and degradation of p53. Biochem J. 2012; 444: 303-14.

27. Lin KY, Tai C, Hsu JC, Li CF, Fang CL, Lai HC, et al. Overexpression of nuclear protein kinase CK2 alpha catalytic subunit (CK2alpha) as a poor prognosticator in human colorectal cancer. PLoS One. 2011; 6: e17193.

28. van Wyk HC, Roxburgh CS, Horgan PG, Foulis AF, McMillan DC. The detection and role of lymphatic and blood vessel invasion in predicting survival in patients with node negative operable primary colorectal cancer. Crit Rev Oncol Hematol. 2014; 90: 77-90.

29. Sun XF, Zhang H. Clinicopathological significance of stromal variables: angiogenesis, lymphangiogenesis, inflammatory infiltration, MMP and PINCH in colorectal carcinomas. Mol Cancer. 2006; 5: 43.

30. Poeschl EM, Pollheimer MJ, Kornprat P, Lindtner RA, Schlemmer A, Rehak P, et al. Perineural invasion: correlation with aggressive phenotype and independent prognostic variable in both colon and rectum cancer. J Clin Oncol. 2010; 28: e358-60.

31. Liebig C, Ayala G, Wilks J, Verstovsek G, Liu H, Agarwal N, et al. Perineural invasion is an independent predictor of outcome in colorectal cancer. J Clin Oncol. 2009; 27: 5131-7. 